\title{
Evaluación de la densidad de siembra y nivel de fertilización en arroz, para las variedades Palmar-18, Lazarroz FL y NayuribeB FL, en Parrita (Pacífico Central), Costa Rica
}

\section{Evaluation of planting density and level of fertilization in rice, for the varieties Palmar-18, Lazarroz FL and NayuribeB FL, in Parrita (central Pacific), Costa Rica}

Juan Gabriel Arias-Badilla1', Edwin Antonio Esquivel-Segura², Rooel Campos-Rodríguez ${ }^{3}$

Arias-Badilla, J; Esquivel-Segura, E; Campos-Rodríguez, R. Evaluación de la densidad de siembra y nivel de fertilización en arroz, para las variedades Palmar-18, Lazarroz FL y NayuribeB FL, en Parrita (Pacífico Central), Costa Rica. Tecnología en Marcha. Vol. 33-3. Julio-Setiembre 2020. Pág 13-24.

doi https://doi.org/10.18845/tm.v33i3.4363

Fecha de recepción: 21 de julio de 2019 Fecha de aprobación: 16 de octubre de 2019

1 Estudiante de maestría en Gestión de Recursos Naturales y Tecnologías de Producción. Instituto Tecnológico de Costa Rica. Costa Rica.

Correo electrónico: Juan_AB17@hotmail.com

2 Doctor en Ciencias Forestales. Instituto Tecnológico de Costa Rica. Costa Rica. Correo electrónico: eesquivel@tec.ac.cr (iD https://orcid.org/0000-0001-9553-060X

3 Doctor en Ciencias Naturales para el Desarrollo. Coordinador del Área Académica Agroforestal. Escuela de Agronegocios, Instituto Tecnológico de Costa Rica. Costa Rica. Correo electrónico: rocampos@tec.ac.cr (D) https://orcid.org/0000-0003-4460-2313 


\title{
Palabras clave
}

Oryza sativa L.; fertilización con NPK; nutrición; rendimiento; arroz en secano.

\section{Resumen}

Los materiales liberados en Costa Rica a la fecha para el cultivo de arroz (Oryza sativa L.) no reciben una distinción en el manejo por parte de los productores del Pacífico Central y Zona Sur de Costa Rica, a pesar de la alta variabilidad del nivel de macollamiento y de la capacidad productiva. Para determinar el efecto de diferentes densidades de siembra y diferentes niveles de fertilización sobre el rendimiento de arroz, se realizó un ensayo en finca La Ligia, en Parrita, Puntarenas. El diseño experimental fue de bloques completos al azar con tratamientos y tres repeticiones para cada una de las variedades (Palmar 18, Lazarroz FL y NayuribeB FL); los tratamientos consistieron en tres densidades de siembra (75, 115 y $155 \mathrm{~kg} / \mathrm{ha}$ de semilla) y 3 niveles de fertilización de N-P-K (50-50-50, 100-50-0, 100-50-100 kg/ha). Las variables evaluadas fueron rendimiento (ton/ha SL), número de macollos / planta, número de panículas / $\mathrm{m}^{2}$, cantidad de granos llenos y peso de 1000 granos; todo lo anterior evaluado en la cosecha del I ciclo de secano correspondiente al año 2017. Para todos los tratamientos se encontraron respuestas significativas. La densidad de siembra de 3,37 qq / ha de semilla favoreció los mejores rendimientos para todas las variedades. Estas además mostraron mejor rendimiento luego de suministrar 100-50-100 kg/ha de fertilizante, y Lazarroz FL fue la variedad que alcanzó los mejores rendimientos (ton/ha SL), seguida de NayuribeB FL y de Palmar 18.

\section{Keywords}

Oryza sativa L.; fertilization with NPK; nutrition; yield; rice in dry land.

\begin{abstract}
The materials released in Costa Rica for rice cultivation (Oryza sativa L.) to date do not receive a differentiated management from producers of the central Pacific and south zone of Costa Rica, despite the high variability in the level of tillering and the productive capacity. To determine the effect of different sowing densities and different levels of fertilization on the yield of rice (Oryza sativa L.) an assay was carried out in the farm La Ligia, which is owned by the company DAPASA, in Parrita, Puntarenas. The experimental design was randomized, of complete blocks with nine treatments and three repetitions, one for each of the varieties (Palmar 18, Lazarroz FL and NayuribeB FL). The treatments compared three planting densities (75, 115, and $155 \mathrm{k}$ seed / ha) and 3 fertilization levels of N-P-K (50-50-50, 100-50-0, 100-50-100 kg / ha). The variables evaluated were yield (ton / ha SL), number of tillers / plants, number of panicles / $\mathrm{m}^{2}$, number of full grains, and weight of 1000 grains, all of them evaluated in the harvest of the 1st dry season in year 2017. For all treatments, significant findings were achieved. Planting density of $155 \mathrm{~kg}$ seed / ha allowed the best yields for the three varieties. Crop yield in every variety was improved after 100-50-100 kg fertilizer / ha was supplied. Lazarroz FL yielded at highest rates (ton / ha SL), followed by NayuribeB FL and Palmar 18.
\end{abstract}

\section{Introducción}

Costa Rica dentro de sus políticas sociales ha buscado combatir la pobreza, presente en mayor medida en áreas rurales y fronterizas [1]. Un pilar fundamental en la alimentación de esta población es el arroz. 
Para el año 2014, en el Censo Agropecuario se reportaron más de 58000 hectáreas plantadas de arroz, de las cuales solo se cosecharon poco más de 52000 hectáreas [2]. El promedio nacional de producción en el periodo 2016-2017 fue de 4,32 toneladas por hectárea; comparativamente, Argentina obtuvo un promedio de 6,66 toneladas por hectárea. Esto obligó al sector arrocero a continuar trabajando en el mejoramiento de variedades y a ser más eficiente en el uso de los insumos y recursos con el fin de generar un menor impacto ambiental [3].

Uno de los aspectos básicos a tomar en cuenta en el cultivo del arroz es la densidad de siembra. Algunas investigaciones sugieren que las densidades más apropiadas para el cultivo de arroz pueden rondar alrededor de los 2,0 a los 2,5 quintales de semilla por hectárea [4].

Las variedades utilizadas en el país difieren en expresión, comportamiento, desarrollo y producción de las variedades tradicionalmente sembradas. La Corporación Arrocera Nacional (CONARROZ) señala que la principal variedad sembrada en el país es Palmar 18, y que variedades más recientes como Lazarroz FL y NayuribeB FL han tomado importancia, ya que para el periodo 2015-2016 se sembraron más de 4000 hectáreas [5].

La fertilización y nutrición en el cultivo de arroz es un tema ampliamente abarcado. En Costa Rica uno de los principales problemas que existen es el análisis de la nutrición vegetal de forma aislada del entorno productivo, por parte del encargado o dueño de la finca. La rentabilidad del productor en el negocio está en función de la capacidad de poder suministrar los insumos adecuados, en el momento más oportuno y bajo el esquema de gastos mínimos y máxima eficiencia. De lo contrario se estaría promoviendo un bajo rendimiento con un alto costo; esta baja rentabilidad podría atentar contra la permanencia del productor en la actividad arrocera. Las recomendaciones de fertilización se basan en rangos de $100 \mathrm{~kg}$ de N, 40 a $60 \mathrm{~kg}$ de P y 80 a $90 \mathrm{~kg}$ de K [6].

Debido a la inexistencia de planes de fertilización específicos actualmente para cada variedad, para el año 2030 la frontera agrícola mundial se habrá expandido en 19 millones de hectáreas, en su mayoría tierras de labranza en secano, por lo que los productores requerirán de un mayor conocimiento de sus variedades [7].

Por la razón indicada, la investigación que aquí se presenta se propuso encontrar la mejor combinación entre fertilización y densidades de siembra para las variedades Palmar 18, Lazarroz FL y NayuribeB FL, con el fin de alcanzar la mayor productividad del cultivo mediante un plan de manejo.

\section{Metodología}

La investigación se desarrolló en condiciones de secano, en la finca La Ligia, Parrita, a una altura de 75 m.s.n.m. [8]. El sitio se ubica dentro de una zona de vida de bosque húmedo tropical. La clasificación del tipo de suelo es Haplic Gleysol del orden Entisol, suborden Orthents [9].

De acuerdo con los datos agroclimáticos históricos de los últimos veinte años en finca La Ligia, la zona Pacífico central cuenta con condiciones favorables para el cultivo de arroz en secano.

En esta investigación se evaluó el comportamiento de las variedades Palmar-18, Lazarroz FL y NayuribeB FL frente a la interacción entre tres densidades de siembra y tres niveles de fertilización (cuadro 1), la cual se concretó en 9 tratamientos con 3 repeticiones, para alcanzar 27 unidades experimentales por cada variedad evaluada; el ensayo en total estuvo conformado por 81 unidades experimentales.

Cada parcela contó con un área de 2,5 $\mathrm{m}^{2}$ de modo que las parcelas estaban separadas entre sí por una franja sin cultivar de $30 \mathrm{~cm}$ con el fin de evitar la interferencia entre tratamientos. El área total del ensayo fue de 423,2 $\mathrm{m}^{2}$. 
La densidad de siembra se estableció en el momento de sembrar (figura 1) y el nivel de fertilización se adecuó a cada tratamiento en los primeros 35 días después de la siembra. Los tratamientos de fertilización (nitrógeno-fósforo-potasio) se practicaron así: en el caso del nivel 50-50-50, se agregó todo el nitrógeno en el momento de la siembra; en el caso del tratamiento 100-50-0 se agregó el 20\% del nitrógeno en el momento de la siembra y el $80 \%$ restante, entre los 15 y los 35 días después de la siembra; en el caso del tratamiento 100-50-100, se agregó un 20\% en el momento de la siembra, se adicionó un 30\% a los 8 días de haber sembrado y se agregó el restante $50 \%$ entre los primeros 15 y 35 días (cuadro 1). Para los tres modalidades de tratamiento, el fósforo y el potasio totales se incorporaron en el momento de la siembra con el fin de aumentar el aprovechamiento y disponibilidad de los nutrientes, disminuyendo así las pérdidas por volatilización en el caso del nitrógeno (cuadro 2).

Cuadro 1. Descripción de los tratamientos del ensayo, finca La Ligia, Parrita, 2017

\begin{tabular}{|c|c|c|c|c|}
\hline \multirow{2}{*}{ Tratamiento } & Densidad de siembra & \multicolumn{3}{|c|}{ Dosis de fertilizante (kg/ha) } \\
\hline & $(\mathrm{qq} / \mathrm{ha})$ & $\mathrm{N}$ & $\mathrm{P}$ & $\mathrm{K}$ \\
\hline 1 & 1,60 & 50 & 50 & 50 \\
\hline 2 & 1,60 & 100 & 50 & 0 \\
\hline 3 & 1,60 & 100 & 50 & 100 \\
\hline 4 & 2,50 & 50 & 50 & 50 \\
\hline 5 & 2,50 & 100 & 50 & 0 \\
\hline 6 & 2,50 & 100 & 50 & 100 \\
\hline 7 & 3,37 & 50 & 50 & 50 \\
\hline 8 & 3,37 & 100 & 50 & 0 \\
\hline 9 & 3,37 & 100 & 50 & 100 \\
\hline
\end{tabular}

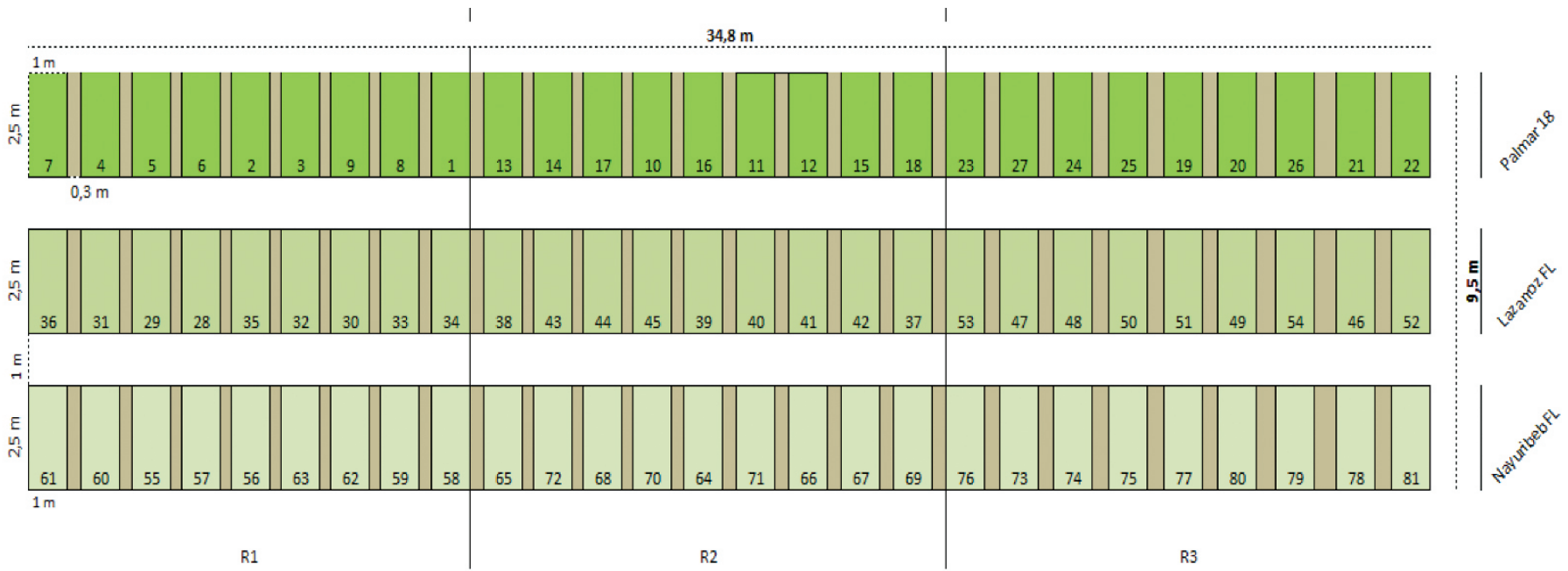

Figura 1. Distribución de bloques al azar, de los tratamientos en el campo (La Ligia, Parrita, 2017) 
Cuadro 2. Programa de fertilización de acuerdo con la edad fisiológica de la planta, la fórmula empleada y el momento de aplicación (La Ligia, Parrita, 2017)

\begin{tabular}{|c|c|c|c|c|c|c|c|c|c|c|c|}
\hline \multirow{3}{*}{ Fecha } & \multirow{3}{*}{$d d s^{*}$} & \multirow{3}{*}{ Tratamiento } & \multicolumn{3}{|c|}{ Aplicación de la fórmula } & \multirow{2}{*}{\multicolumn{6}{|c|}{ Aporte elemento (kg/ha) }} \\
\hline & & & \multirow{2}{*}{ Fórmula } & \multirow{2}{*}{$\begin{array}{l}\text { Cantidad } \\
(\mathrm{qq} / \mathrm{ha})^{\star}\end{array}$} & \multirow{2}{*}{$\begin{array}{c}\text { Cantidad } \\
\text { (kg/ha) }\end{array}$} & & & & & & \\
\hline & & & & & & $\mathrm{N}^{*}$ & $P$ & K & $\mathrm{Mg}$ & $S$ & $\mathrm{~B}$ \\
\hline $11 / 7 / 2017$ & 0 & $50-50-50$ & $26-0-26$ & 1,00 & 46,00 & 12,0 & 0,0 & 12,0 & 0,0 & 0,0 & 0,0 \\
\hline $11 / 7 / 2017$ & 0 & $50-50-50$ & $18-46-0$ & 2,37 & 109,02 & 19,6 & 50,1 & 0,0 & 0,0 & 0,0 & 0,0 \\
\hline $11 / 7 / 2017$ & 0 & $50-50-50$ & $\mathrm{KCl} 60 \%$ & 1,37 & 63,02 & 0,0 & 0,0 & 37,8 & 0,0 & 0,0 & 0,0 \\
\hline $11 / 7 / 2017$ & 0 & $50-50-50$ & $\begin{array}{c}\text { Urea } \\
40 \%+7,7(S)\end{array}$ & 1,00 & 46,00 & 18,4 & 0,0 & 0,0 & 0,0 & 3,5 & 0,0 \\
\hline $11 / 7 / 2017$ & 0 & $100-50-0$ & $18-46-0$ & 2,37 & 109,02 & 19,6 & 50,1 & 0,0 & 0,0 & 0,0 & 0,0 \\
\hline $26 / 7 / 2017$ & 15 & $100-50-0$ & $\begin{array}{c}\text { Urea } \\
40 \%+7,7(S)\end{array}$ & 2,00 & 92,00 & 36,8 & 0,0 & 0,0 & 0,0 & 7,1 & 0,0 \\
\hline $15 / 8 / 2017$ & 35 & $100-50-0$ & $\begin{array}{c}\text { Urea } \\
40 \%+7,7(S)\end{array}$ & 2,35 & 108,10 & 43,2 & 0,0 & 0,0 & 0,0 & 8,3 & 1,0 \\
\hline $11 / 7 / 2017$ & 0 & $100-50-100$ & $18-46-0$ & 2,36 & 108,56 & 19,5 & 49,9 & 0,0 & 0,0 & 0,0 & 0,0 \\
\hline $11 / 7 / 2017$ & 0 & $100-50-100$ & $\mathrm{KCl} 60 \%$ & 2,50 & 115,00 & 0,0 & 0,0 & 69,0 & 0,0 & 0,0 & 0,0 \\
\hline $19 / 7 / 2017$ & 8 & $100-50-100$ & $26-0-26$ & 2,60 & 119,60 & 31,1 & 0,0 & 31,1 & 0,0 & 0,0 & 0,0 \\
\hline 26/7/2017 & 15 & $100-50-100$ & $\begin{array}{c}\text { Urea } \\
40 \%+7,7(S)\end{array}$ & 1,00 & 46,00 & 18,4 & 0,0 & 0,0 & 0,0 & 3,5 & 0,0 \\
\hline $15 / 8 / 2017$ & 35 & $100-50-100$ & $\begin{array}{c}\text { Urea } \\
40 \%+7,7(S)\end{array}$ & 1,70 & 78,20 & 31,3 & 0,0 & 0,0 & 0,0 & 6,0 & 0,0 \\
\hline
\end{tabular}

* dds: días después de siembra, qq: quintales (1 quintal = $46 \mathrm{~kg}$ )

El manejo nutricional de los tratamientos estuvo dirigido a cubrir las necesidades tempranas del cultivo y a potenciar la capacidad nutricional del suelo, por lo que en el momento de la siembra se agregaron todo el fósforo y todo el potasio asignados a cada tratamiento. Únicamente en el caso del tratamiento 100-50-0, se agregaron 2,6 qq/ha de la fórmula 26-0-26 8 días después de la siembra, para terminar de cubrir el nivel de potasio establecido y a la vez adicionar un 31\% de nitrógeno. A todos los tratamientos se terminaron de aplicar los fertilizantes 10 días antes de la diferenciación del primordio floral, tomando como referencia el ciclo de vida de la variedad Palmar 18.

Para evaluar el nivel productivo de las densidades de siembra que se establecieron, es decir, a 1,60 qq/ha, 2,50 qq/ha, y 3,37 qq/ha, se realizaron las mediciones de laboratorio bajo el reglamento interno para la valoración del arroz en granza según la normativa de CONARROZ, donde se indican las fórmulas ajustadas y estandarizadas para el uso en las industrias arroceras de Costa Rica [10]. Para determinar el rendimiento húmedo y sucio de los tratamientos correspondientes a los tres materiales diferenciados según densidad, se empleó la siguiente formula: 


$$
\text { Rendimiento húmedo y sucio }=\left(\frac{\text { peso neto }}{1000} \times \frac{10000}{\text { área ensayo }}\right) \times 1000
$$

Luego se eliminaron las impurezas, se redujo la humedad a un nivel entre $11,2 \%$ y $12,1 \%$ y se procedió a sacar el rendimiento seco y limpio:

$$
\text { Rendimiento seco y limpio }=\left(\text { Rend } \frac{\mathrm{H}}{\mathrm{S}} \times \frac{(100-\text { humedad inicial })}{(100-13)} \times \frac{(100 \text {-impurezas })}{(100-1,5)}\right)
$$

El ente regulador nacional, CONARROZ, admite un porcentaje de humedad de $13 \%$ y un 1,5\% de impurezas.

Posteriormente se evaluaron por separado los tratamientos a los que se sometió cada material y con base en los rendimientos de grano seco y limpio, se seleccionaron las densidades ideales con las que el productor debería manejar dichas variedades en la región del Pacífico Central [10].

La evaluación de la eficiencia en el uso de fertilizantes nitrogenados para cada variedad se realizó mediante la aplicación de la fórmula:

Eficiencia de fertilización: Rend (mayor) del tratamiento (100-50-100)- Rend (menor) del tratamiento (50-50-50) Dosis de fertilizante $(\mathrm{N})$

Una vez identificados los mejores rendimientos para cada variedad de acuerdo con los tratamientos aplicados, se realizó una comparación entre los resultados de cada uno y se seleccionó el mejor tratamiento tomando en cuenta las condiciones ambientales de Parrita y la interacción entre la densidad de siembra, el nivel de fertilización y el rendimiento productivo. De este modo se generó una guía agronómica sobre el manejo nutricional y la densidad de semilla por área sembrada para las variedades Palmar-18, Lazarroz FL y NayuribeB FL dirigida a los agricultores del Pacífico central.

También se realizó un análisis de varianza (andeva) de cada una de las variedades, así como una prueba de normalidad de Shapiro-Wilk para contrastar cada uno de los tratamientos con los restantes, utilizando el programa estadístico de Infostat. Se utilizó un nivel de significancia $\mathrm{p}<0,05$.

\section{Resultados y discusión}

\section{Evaluación del nivel productivo}

Las variedades Palmar 18, Lazarroz FL y NayuribeB FL en el Pacífico central de Costa Rica resultan afectadas por la densidad de siembra, así como por el nivel de fertilización que se establezca para el cultivo ( $p<0,0001$ ), conforme a los resultados obtenidos. La media de los rendimientos secos y limpios en toneladas por hectárea para cada variedad fue de 3,51 ton/ha para Palmar 18; 4,83 ton/ha para Lazarroz FL, y 4,17 ton/ha para NayuribeB FL (figura 2).

El promedio nacional para la cosecha 2016-2017 fue de 4,32 toneladas de arroz seco y limpio [11], es decir que Palmar 18 y NayuribeB FL no superaron el promedio nacional. En el caso de Lazarroz FL el rendimiento obtenido fue 11,80\% superior al rendimiento promedio nacional, por lo tanto, se puede afirmar que Lazarroz FL es una variedad promisoria en Costa Rica. 


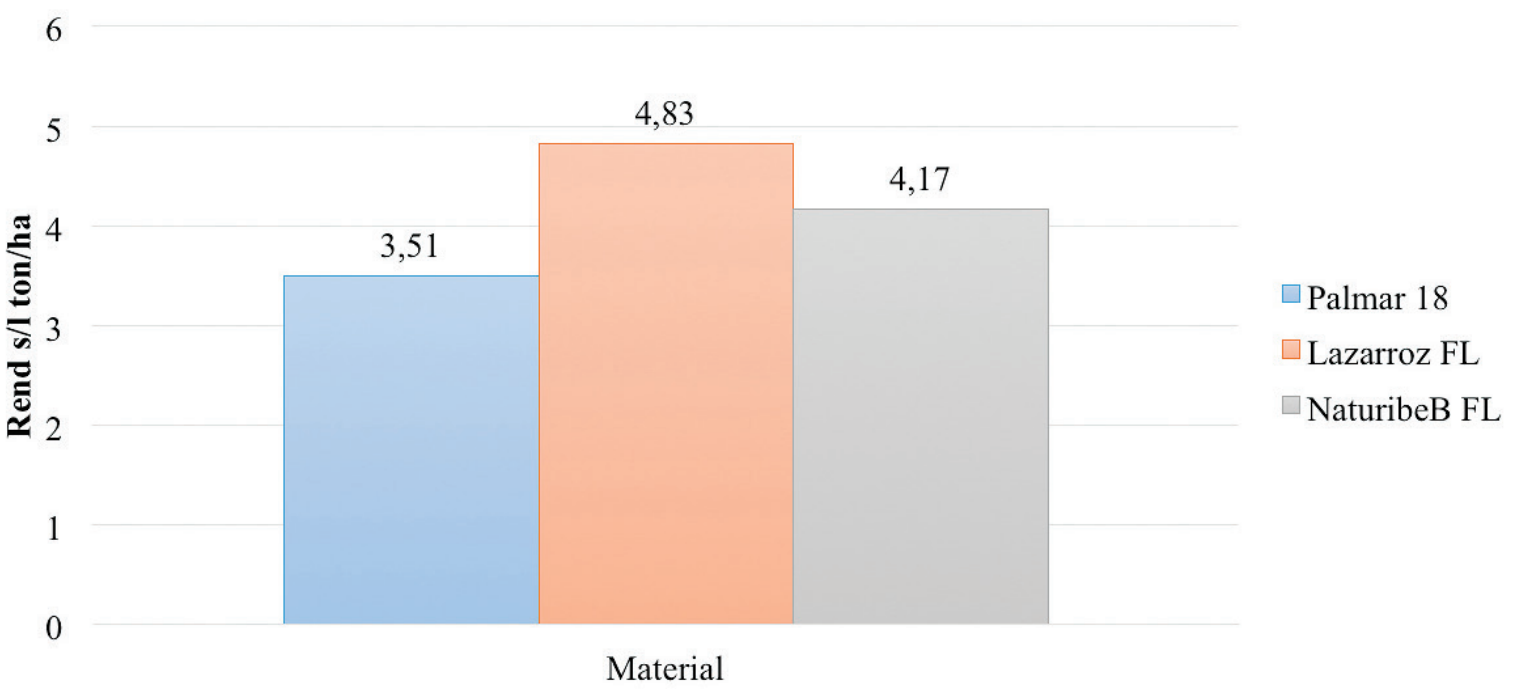

Figura 2. Rendimiento promedio en toneladas por hectárea con base en la densidad de siembra, para las variedades Palmar 18, Lazarroz FL y NayuribeB FL (La Ligia, Parrita, 2017)

Los agricultores en Costa Rica tradicionalmente han empleado densidades de siembra entre 3,5 y 4 quintales por hectárea [12]; sin embargo, esta práctica generalmente propicia el desarrollo de plantas más delgadas y débiles, que son más susceptibles al volcamiento y al ataque de plagas y enfermedades. Las nuevas variedades Palmar 18, Lazarroz FL y principalmente NayuribeB FL, a densidades entre 2,5 y 3,37 quintales por hectárea, propician una mejor respuesta a la fertilización. De acuerdo con lo anterior, se deduce que, pese al potencial genético de las variedades analizadas, el manejo agronómico de la plantación puede incidir en una disminución en la productividad.

La variedad que mostró una mejor respuesta al aumento en la densidad de siembra por hectárea fue Lazarroz FL, ya que superó las 5 toneladas por hectárea (cuadro 4) en promedio a densidades de 2,50 y 3,37 qq/ha, y alcanzó la mejor productividad a 3,37 qq/ha. En el caso de NayuribeB FL, a 2,50 qq/ha logró alcanzar una productividad similar a la obtenida con la variedad anterior a la densidad de 3,37 qq/ha; con esto se deduce que NayuribeB FL es una variedad con mejor capacidad de macollamiento, es decir que a una densidad de 2,50 qq/ha logra generar una cantidad de tallos efectivos mayor que Lazarroz FL y a su vez logra mejores rendimientos por área sembrada que Palmar 18.

\section{Eficiencia en el uso de los fertilizantes nitrogenados}

La deficiencia de nitrógeno afecta la etapa de macollamiento, es decir, el índice de crecimiento de la planta; el fósforo de igual forma afecta el correcto macollamiento, y también puede dañar la panícula si se encuentra en concentraciones tóxicas durante la etapa de maduración [13]. En los tres tratamientos con diferentes niveles de fertilización Lazarroz FL logró ser la variedad más productiva, y se destacó como una variedad muy eficiente, incluso ante bajos niveles de fertilización (cuadro 3).

Los rendimientos siempre fueron mejores cuando se aumentaron tanto el nitrógeno como el Potasio, si bien el suelo presentaba contenidos óptimos de ambos elementos.

Durante el periodo de crecimiento los cultivos absorben grandes cantidades de potasio [14] y en la etapa de madurez, si se encuentra en concentraciones insuficientes, se afectan tanto el macollamiento como la panícula [13], [15]. Por esta razón, al aumentar el suministro de potasio, las tres variedades mostraron una mejora en sus rendimientos (cuadro 4). 
Cuadro 3. Rendimiento promedio de las variedades Palmar 18, Lazarroz FL y NayuribeB FL de acuerdo con los niveles de fertilización 50-50-50, 100-50-0 y 100-50-100 kg/ha (La Ligia, Parrita, 2017)

\begin{tabular}{|c|c|c|c|}
\hline Dosis de fertilizante kg/ha & $50-50-50$ & $100-50-0$ & $100-50-100$ \\
\hline Palmar 18 & 2,52 & 3,57 & 4,46 \\
\hline Lazarroz FL & 3,43 & 4,84 & 6,20 \\
\hline NayuribeB FL & 2,72 & 3,89 & 5,89 \\
\hline
\end{tabular}

Cuadro 4. Rendimiento promedio de las variedades Palmar 18, Lazarroz FL y NayuribeB FL de acuerdo con las interacciones entre las tres densidades de siembra y los tres niveles de fertilización (La Ligia, Parrita, 2017)

\begin{tabular}{|c|c|c|c|c|c|c|c|c|}
\hline & & & \multicolumn{2}{|c|}{ Palmar 18} & \multicolumn{2}{|c|}{ Lazarroz FL } & \multicolumn{2}{|c|}{ NayuribeB FL } \\
\hline $\begin{array}{l}\text { Densidad } \\
\text { desiembra }\end{array}$ & $\begin{array}{l}\text { Dosis de } \\
\text { fertilizante } \mathrm{kg} / \mathrm{ha}\end{array}$ & Tratamiento & \multicolumn{2}{|c|}{ Media } & \multicolumn{2}{|c|}{ Media } & \multicolumn{2}{|c|}{ Media } \\
\hline 1,60 & $50-50-50$ & 1 & 2,00 & $A^{*}$ & 2,7 & $A$ & 2,37 & A \\
\hline 1,60 & $100-50-0$ & 2 & 2,63 & B & 3,10 & $A B$ & 2,93 & B \\
\hline 1,60 & $100-50-100$ & 3 & 3,23 & C & 4,20 & C & 4,30 & C \\
\hline \multicolumn{3}{|c|}{ Promedio } & 2,62 & & 3,33 & & 3,20 & \\
\hline 2,50 & $50-50-50$ & 4 & 2,57 & B & 3,40 & B & 2,70 & $A B$ \\
\hline 2,50 & $100-50-0$ & 5 & 3,77 & D & 5,30 & D & 4,13 & C \\
\hline 2,50 & $100-50-100$ & 6 & 4,87 & F & 6,63 & $\mathrm{~F}$ & 6,57 & D \\
\hline \multicolumn{3}{|c|}{ Promedio } & 3,73 & & 5,11 & & 4,47 & \\
\hline 3,37 & $50-50-50$ & 7 & 3,00 & C & 4,20 & C & 3,10 & B \\
\hline 3,37 & $100-50-0$ & 8 & 4,30 & E & 6,13 & $E$ & 4,60 & C \\
\hline 3,37 & $100-50-100$ & 9 & 5,27 & G & 7,77 & $\mathrm{G}$ & 6,80 & D \\
\hline \multicolumn{3}{|c|}{ Promedio } & 4,19 & & 6,03 & & 4,83 & \\
\hline
\end{tabular}

* Letras distintas muestran diferencias significativas al 0,05 entre los tratamientos.

El mejor rendimiento en los casos de Palmar 18 y Lazarroz FL se alcanzó cuando se sembraron a una densidad de 3,37 qq/ha junto con una dosis de fertilizante de 100-50-100 kg/ha. Es importante destacar que Lazarroz FL alcanzó el mismo rendimiento cuando se emplearon 1,60 qq/ha de semilla junto con la misma dosis de nutrientes, 100-50-100 kg/ha, que cuando se emplearon 3,37quintales de semilla con una dosis de 50-50-50 kg/ha de nutrientes; por tanto, para esta variedad la densidad de siembra es determinante en el rendimiento por alcanzar en campo.

En el caso de NayuribeB FL se obtuvieron rendimientos similares cuando se sembró a 2,50 y a 3,37 qq/ha de semilla junto con 100-50-100 kg/ha de nutrientes.

Rodríguez [16], Meléndez y Molina [17], el Instituto Internacional de Nutrición de Plantas (IPNI, por sus siglas en inglés) [18] y Chaudhary et al. [13] coinciden en que la cantidad de los 
nutrientes necesarios en los trópicos para producir una tonelada de arroz en cáscara es de $20,5 \mathrm{~kg}$ de nitrógeno; 5,1 kg de fósforo, y 44,4 kg de potasio por hectárea. De acuerdo con las medias de cada variedad y su relación con la eficiencia en el uso de los nutrientes, se puede afirmar que la dosis 100-50-100 kg/ha suministrada favoreció a cada una de las variedades y su rendimiento por hectárea.

La eficiencia en el uso de los fertilizantes corresponde a la cantidad de producto cosechado por la unidad de nutrientes aplicada al suelo [19], por lo tanto, cuando se logran mayores rendimientos siempre utilizando la misma cantidad de fertilizante, se ha logrado alcanzar una mayor eficiencia.

La evaluación de la eficiencia de la fertilización nitrogenada, en el caso de Palmar 18, dio como resultado:

Eficiencia de fertilización: $\frac{5270 \mathrm{~kg}-2000 \mathrm{~kg}}{100}=32,7 \mathrm{~kg} \cdot$ ha-1 de grano por $\mathrm{kg}$ de nitrógeno

Es decir, por cada kg por hectárea agregado de nitrógeno, la eficiencia de conversión de la planta fue de 32,7 kg de grano; la eficiencia claramente fue mayor en NayuribeB FL, 44,3 y en Lazarroz FL, 50,7.

Cuadro 5. Valor de p para cada una de las variedades analizadas y su interacción con ellas (La Ligia, Parrita, 2017)

\begin{tabular}{|c|c|c|c|}
\hline \multirow{2}{*}{ Variable } & \multicolumn{3}{|c|}{$p$-valor } \\
\hline & Palmar 18 & Lazarroz FL & NayuribeB FL \\
\hline Conteo de tallos & 0,4009 & 0,6355 & 0,2697 \\
\hline Tallos efectivos & 0,1452 & 0,3256 & 0,0992 \\
\hline Longitud de panícula & 0,0016 & 0,0068 & $凶 0,0001$ \\
\hline Cantidad de granos llenos & 0,0846 & 0,0005 & 0,0040 \\
\hline Peso de 1000 granos & 0,0505 & 0,0012 & 0,0025 \\
\hline
\end{tabular}

Las deficiencias de nitrógeno así como las deficiencias de fósforo reducen la capacidad de macollamiento de las plantas de arroz [15]. A su vez, las plantas con deficiencia de fósforo presentan tallos más delgados y alargados, siendo a su vez plantas más pequeñas y de crecimiento más retardado. De acuerdo con lo anterior, se determina una concordancia entre la formación deficiente de tallos efectivos y la cantidad de granos llenos para el caso de Palmar 18. El conteo de tallos y los tallos efectivos no mostraron relación con la cantidad de nutrientes suministrada; por lo contrario, la longitud de panícula, la cantidad de granos llenos y el peso de 1000 granos mostraron una respuesta positiva $(p<0,001)$ ante el suministro de nutrientes (cuadro 5).

Plan de manejo de la densidad de siembra y el nivel de fertilización

La mayor demanda nutricional en el cultivo de arroz corresponde a los elementos nitrógeno, fósforo y potasio, y su nivel de disponibilidad va a depender de las condiciones del suelo, así como del mismo manejo del cultivo [20]. 


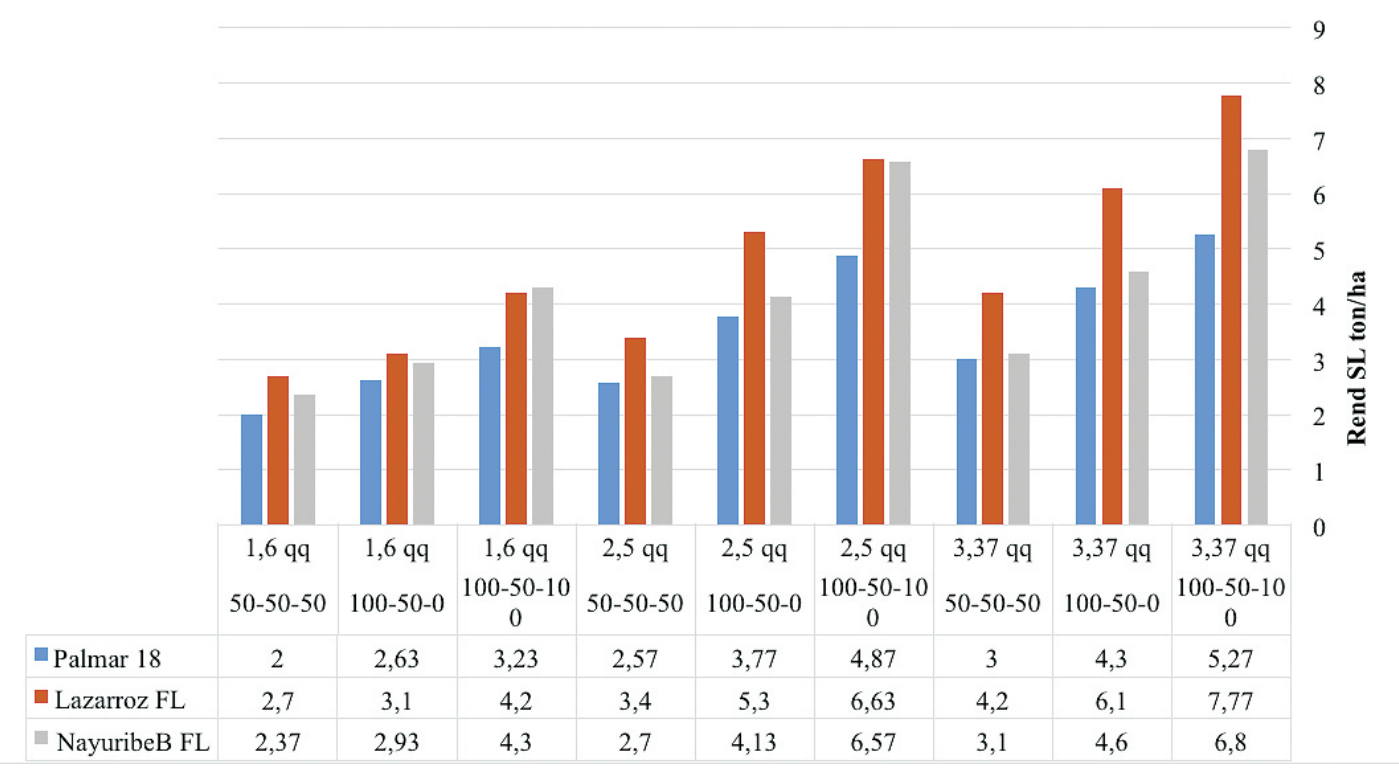

Figura 3. Rendimiento en toneladas por hectárea e interacción entre el nivel de fertilización y la densidad de siembra para las variedades Palmar 18, Lazarroz FL y NayuribeB FL (La Ligia, Parrita, 2017)

De acuerdo con el rendimiento en toneladas por hectárea, se puede afirmar que las tres variedades alcanzaron los mejores rendimientos cuando se utilizó el programa con 100-50$100 \mathrm{~kg}$ de nutrientes por hectárea. En la figura 3 se puede observar con mayor claridad el rendimiento de cada variedad de acuerdo con la interacción entre la densidad de siembra y el nivel de fertilización.

Los niveles de extracción y eficiencia de la fertilización varían entre regiones y principalmente entre variedades, ya que cada variedad tiene una eficiencia específica en la absorción de nutrientes. Los niveles de eficiencia de la fertilización en esta investigación superan los reportados por Molina y Rodríguez [20], lo cual se debe principalmente a la capacidad de las tres variedades, principalmente de Lazarroz FL, de sintetizar el nitrógeno asimilable del medio.

Con respecto a la densidad de siembra, la variedad Palmar 18 mostró una gran adaptabilidad a la zona en cuanto a constancia productiva, sin importar la densidad de siembra a la que se estableció el cultivo. En el caso de Palmar 18, la mejor densidad de siembra se encontró entre 2,5 y 3,37 qq/ha. Dentro de este margen la variedad se va a desempeñar satisfactoriamente.

La variedad Lazarroz FL mostró ser mucho más dependiente de la densidad de siembra que las otras. Cuando se sembró Lazarroz FL a 1,6 qq/ha, los rendimientos nunca superaron las 4 ton/ ha. Cuando se sembró a 2,5 qq/ha, el rendimiento aumentó considerablemente; sin embargo, los mejores rendimientos con esta variedad se alcanzaron solo cuando se sembró a 3,37 qq/ ha (cuadro 6).

NayuribeB FL en todos los ensayos, sin importar la densidad de siembra, estuvo por encima de Palmar 18; si se sembrara a densidades entre 2,5 y 3,37 qq/ha, se esperarían rendimientos similares a los obtenidos con Lazarroz FL. Estos resultados van a depender de las condiciones del suelo, ambientales y del manejo (cuadro 6).

Los mejores rendimientos de Palmar 18 se obtuvieron cuando se sembró a densidades superiores a 2,5 qq/ha y con una fertilización de $100 \mathrm{~kg} \mathrm{~N}-50 \mathrm{~kg}$ P - $100 \mathrm{~kg} \mathrm{~K}$. ParPalmar 18 en secano, la planta aún podría tolerar más kilogramos de nitrógeno por hectárea, sin sobrepasar los $120 \mathrm{~kg} / \mathrm{ha}$, ya que los tallos pueden ceder y volcarse. 
Cuadro 6. Especificaciones técnicas para las variedades Palmar 18, Lazarroz FL y NayuribeB FL (La Ligia, Parrita)

\begin{tabular}{|c|c|c|c|c|}
\hline \multirow{2}{*}{ Variedad } & Densidad de siembra & \multicolumn{3}{|c|}{ Dosis nutricionales sugeridas } \\
\cline { 3 - 5 } & sugerida (qq/ha) & $N$ & $P$ & $\mathrm{~K}$ \\
\hline Palmar 18 & $2,5-3,37$ & $50-100$ & 50 & $50-100$ \\
\hline Lazarroz FL & $2,5-3,37$ & $50-100$ & 50 & $50-100$ \\
\hline NayuribeB FL & $2,5-3,37$ & $50-100$ & 50 & $50-100$ \\
\hline
\end{tabular}

Los mejores rendimientos de Palmar 18 se obtuvieron cuando se sembró a densidades superiores a 2,5 qq/ha y con una fertilización de $100 \mathrm{~kg} \mathrm{~N}-50 \mathrm{~kg}$ P - $100 \mathrm{~kg} \mathrm{~K}$. Para Palmar 18 en secano, la planta aún podría tolerar más kilogramos de nitrógeno por hectárea, sin sobrepasar los $120 \mathrm{~kg} / \mathrm{ha}$, ya que los tallos pueden ceder y volcarse.

Lazarroz FL es una variedad con capacidad para aprovechar el nitrógeno ambiental por esta razón, a todas las dosis proporcionadas, se logró alcanzar muy buenos rendimientos. El tratamiento con 100-50-100 kg/ha fue el mejor. Para este material no se recomienda sobrepasar los $100 \mathrm{~kg}$ de nitrógeno / ha aportado mediante fertilizantes. Esta variedad tiene que sembrarse a densidades no menores de 2,5 qq de semilla / ha, esto para minimizar los problemas de manejo en la finca y para aumentar los rendimientos productivos por hectárea.

La variedad NayuribeB FL cuenta con una alta capacidad de macollamiento, por lo que se sugiere manejarla en secano con densidades entre 2,50 y 3,37 qq/ha y no sobrepasar estas densidades porque se podría incidir en una disminución de los granos llenos por panícula y en la cantidad de panículas por área sembrada. Nutricionalmente NayuribeB FL en secano tolera niveles de fertilización superiores a Lazarroz FL; por lo tanto, se recomienda manejar una fertilización igual a $100 \mathrm{~kg}$ de nitrógeno / ha y aumentar la cantidad de potasio, para propiciar un buen crecimiento de panículas y, por consiguiente, un mayor rendimiento del cultivo.

\section{Conclusiones}

Las tres variedades evaluadas alcanzaron su máxima productividad cuando se sembraron a una densidad de 3,37 qq/ha; a su vez, NayuribeB FL no mostró diferencias significativas con ese resultado al sembrarla a 2,50 qq/ha. También se observó que el desempeño de las variedades depende más del vigor genético que de la cantidad de semilla sembrada. NayuribeB FL, sin importar el nivel de fertilización, generó mayor cantidad de tallos efectivos que Lazarroz FL.

Las tres variedades mostraron mayor rendimiento al aumentar el suministro de nitrógeno y potasio. Lazarroz FL fue la variedad más eficiente en aprovechamiento del nitrógeno, además de la más productiva.

En los tratamientos en que se buscó hacer un manejo que generara menor impacto ambiental, es decir, incorporar la menor cantidad de nutrientes a la siembra, se obtuvieron plantas de menor vigor, lo cual se tradujo en menor floración y, por consiguiente, menor rendimiento.

En el Pacífico central de Costa Rica, Palmar 18 y NayuribeB Fl se pueden cultivar a densidades iguales o superiores a 2,50 qq/ha; Lazarroz FL requiere ser cultivado a 3,37 qq/ha para mostrar su potencial productivo. El nivel de fertilización para todas las variedades debe ser igual 0 superior a $100 \mathrm{~kg}$ de nitrógeno, al mismo tiempo que debe realizarse un suministro de potasio, y de fósforo cercano o igual a $50 \mathrm{~kg}$; todos los requerimientos van a depender de la concentración de los nutrientes en el suelo. 


\section{Referencias}

[1] Organización de las Naciones Unidas para la Alimentación (FAO), "Hacia la elaboración de una estrategia de asistencia técnica de la FAO en apoyo a la implementación de la Iniciativa América Latina y el Caribe Sin Hambre", Ciudad de Guatemala, 2016.

[2] Instituto Nacional de Estadística y Censos (INEC), "VI Censo Nacional Agropecuario; resultados generales", 2014 [en línea]. Disponible en http://www.mag.go.cr/bibliotecavirtual/a00338.pdf.

[3] M. Acevedo Barona et al., "Efectos de la densidad de siembra y fertilización nitrogenada sobre el rendimiento de granos de arroz del cultivar centauro en Venezuela", Agronomía Tropical, vol. 61, no. 1, pp. 15-26, 2011.

[4] R. Tinoco y A. Acuña, "Manual de recomendaciones técnicas del cultivo de arroz (Oryza sativa)", San José, Costa Rica: INTA, 2008 [en línea]. Disponible en http://www.mag.go.cr/bibliotecavirtual/a00177.pdf.

[5] CONARROZ, "Informe estadístico periodo 2016/2017", Unidad de Inteligencia de Mercados, Dirección de Operaciones, Costa Rica, 2016 [en línea]. Disponible enhttps://www.conarroz.com/UserFiles/File/INFORME_ ANUAL_ESTADISTICO_PERIODO_2016_2017.pdf

[6] D. Guzmán, "Manejo agronómico del cultivo de arroz (Oryza sativa L.) sembrado bajo riego en finca Ranchos Horizonte", Cañas, Guanacaste, Costa Rica, pp. 6-8, 2006.

[7] FAO, "Agricultura mundial hacia los años 2015/2030", Roma, Italia, 2002 [en línea]. Disponible en http://www. fao.org/docrep/004/y3557s/y3557s00.htm\#TopOfPage

[8] H. Jiménez, "Anatomía del sistema de clasificación de Holdridge", Turrialba, Costa Rica, 1982.

[9] R. Mata, A. Vásquez y A. Rosales, "El mapa de suelos de Costa Rica con leyenda WRB", 2016.

[10] CONARROZ, "Reglamento interno del mecanismo para la valoración del arroz en granza", 2004 [en línea]. Disponible en http://www.conarroz.com/UserFiles/File/Transparencia/ MECANISMOparaLaVALORACIONdeIARROZenGRANZAparaCOSTA\%2ORICA.pdf

[11] CONARROZ, "Informe estadístico período 2016/2017", Unidad de Inteligencia de Mercados, Dirección de Operaciones, 2017.

[12] CONARROZ, "Factores claves en el manejo de arroz de secano", San José, Costa Rica, 2005 [en línea]. Disponible en http://www.conarroz.com/pdf/Factores\%20claves\%20en\%20el\%20manejo\%20de\%20arroz\%20 de\%20secano.pdf

[13] R.C. Chaudhary, J. S. Nanda y D.V. Tran, "Guía para identificar las limitaciones de campo en la producción de arroz", FAO, Roma, Italia, 2003.

[15] Potash \& Phosphate Institute, "Identificación de los problemas nutricionales en arroz", 2017 [en línea]. Disponible en https://ipni.net/ppiweb/gltamn.nst/\$webindex/E4B79420325A209705256ECB004BDE01?opend ocument\&print=1

[16] J. Rodríguez, "Fertilización del cultivo de arroz (Oryza sativa L.)", XI Congreso Nacional Agronómico, pp. 1-14, 1999.

[17] G. Meléndez y E. Molina, "Fertilidad de suelos y manejo de la nutrición de cultivos en Costa Rica", Centro de Investigaciones Agronómicas, pp. 92-103, 2001.

[18] Instituto Internacional de Nutrición de Plantas, "Requerimientos nutricionales de los cultivos", Archivo Agronómico no. 3, 2002 [en línea]. Disponible enhttp://lacs.ipni.net/topic/agronomic-files.

[19] R. Ramírez, "El uso eficiente de los fertilizantes y el incremento de la productividad agrícola en Venezuela", Agronomía Tropical, vol. 40, 1990 [en línea]. Disponible en http://www.sian.inia.gob.ve/revistas_ci/ Agronomia\%20Tropical/at4013/pdf/ramirez_r.pdf

[20] E. Molina y J. Rodríguez, "Fertilización con N, P, K y S, y curvas de absorción de nutrimentos en arroz var cfx. 18 en Guanacaste", Agronomía Costarricense, 2012. 